\title{
CONOSCOPIC HOLOGRAMS
}

\author{
Gabriel Y. SIRAT \\ Ecole Nationale Superieure de Telecommunications, Optique des Materiaux, 46 rue Barrault, 75634 Paris Cedex 13, France \\ and \\ Demetri PSALTIS \\ Department of Electrical Engineering, California Institute of Technology, Pasadena, CA 91125, USA
}

Received 21 August 1987

Conoscopic holography is an incoherent method for optically recording three dimensional objects using light propagation in birefrigent crystals. Three separate methods for reconstructing conoscopic holograms are presented and experimentally demonstrated.

\section{Introduction}

In a recent paper we introduced a simple method for holographic recording based on light propagation in uniaxial crystals [1]. In such crystals the birefrigence induces an angular dependence of the extraordinary index of refraction causing an angle dependent change in the optical path. When a beam of light passes through a birefrigent crystal and it is viewed through a pair of crossed polarizers, the resulting interference patterns are called conoscopic figures. The conoscopic figure that results at a plane perpendicular to the optical axis from a single monochromatic point source is a sinusoidal Fresnel zone lens (FZL). The optical set up for recording the conoscopic figure of a single point source is shown in fig. 1 and the resulting FZL is shown in fig. 2. The conoscopic figure shown in fig. 2 was obtained using a circular polarizer and analyzer and this eliminates the cross pattern that is superimposed on the FZL when linear polarizers are used. The conoscopic figure can be formed either with coherent light or alternatively with quasimonochromatic spatially incoherent light.

The center of the FZL is a projection of the source point on the observation plane and its focal length is proportional to the distance between the point

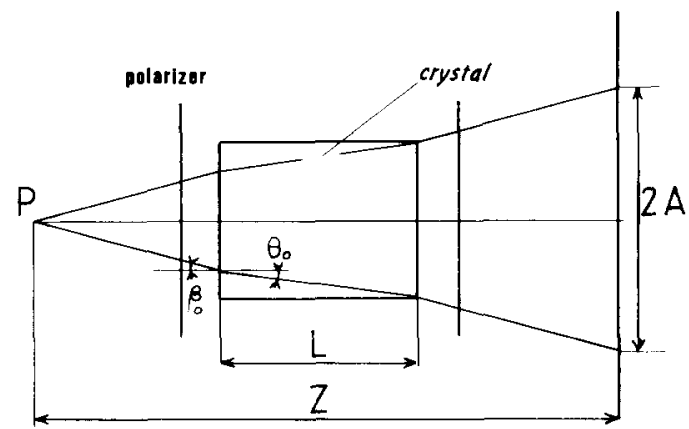

Fig. 1. Principle of conoscopic holography.

source and the observation plane. The formation of the conoscopic FZL can be thought of as a hologram of the point source since sufficient information about its position in all three dimensions is preserved. The formation of the conoscopic hologram is very similar to recording a Fresnel hologram of a point source that is formed interferometrically with coherent light. Note however that the conoscopic hologram can be formed with spatially incoherent light. We can also choose to think of the formation of the conoscopic hologram as an interferometric process, whereby the ordinary and extraordinary waves interfere after the analyzer. Since the geometrical paths of these two 


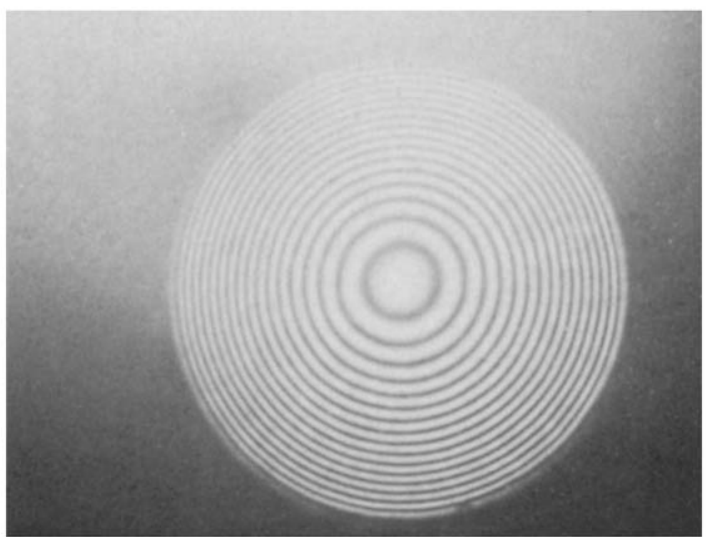

Fig. 2. Experimental transfer function of the system.

waves are identical, spatial coherence is not required for interference to occur.

An arbitrary object can be viewed as a collection of points sources. Thus the conoscopic pattern of an arbitrary object is a linear superposition of FZL's as shown in fig. 3. The recorded FZL's contain all the information that is necessary for reconstructing the 3-D object and is thus a conoscopic hologram of the object. Shown in figs. $4 \mathrm{a}$ and $4 \mathrm{~b}$ are the conoscopic holograms of two point sources and a transparency of the word "FOURIER" respectively. The crystal used to form these conoscopic holograms was calcite grade B with dimensions $40 \mathrm{~mm}(x) \times 40 \mathrm{~mm}(y) \times$ $38 \mathrm{~mm}(z)$ and the light source was a sodium lamp. A photograph of the crystal and a schematic diagram of the optical set up are shown in fig. 5. There are three methods for reconstructing conoscopic holograms that will be discussed: conoscopic, diffraction, and computer reconstructions. In the remainder of the paper we present an experimental demonstration of each of these three methods.

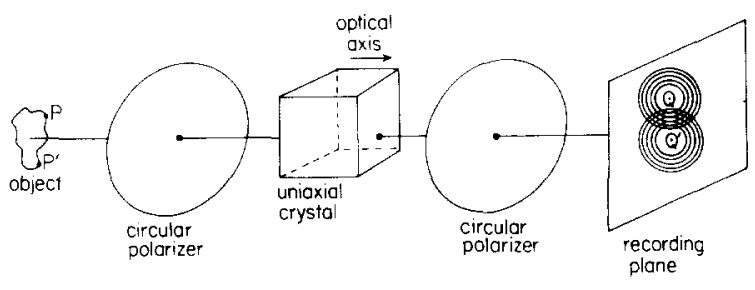

Fig. 3. Optical set-up.
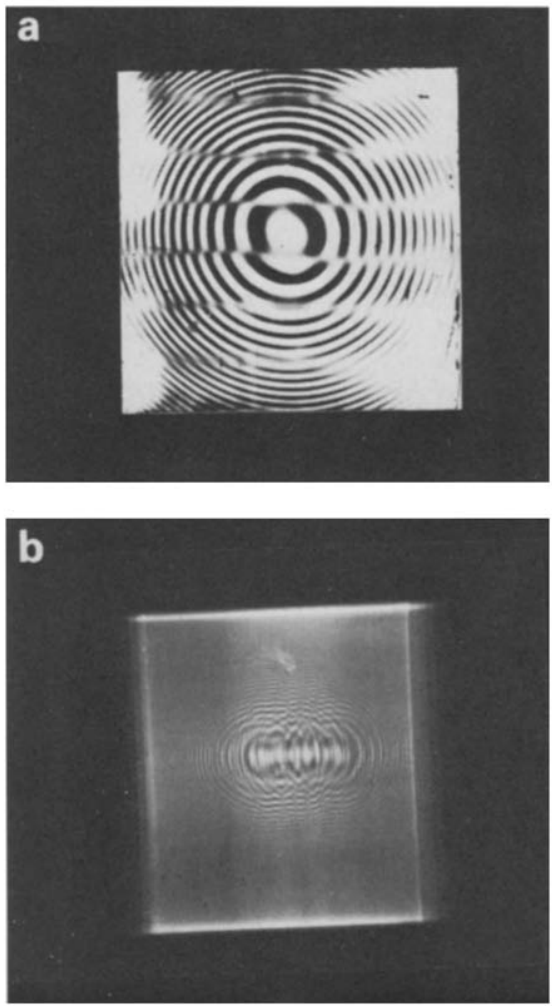

Fig. 4. Conoscopic holograms of two points (a), and of the word "FOURIER" (b).

\section{Conoscopic reconstruction}

The same apparatus that is used to record a conoscopic hologram can also be used for reconstruction. In reference to fig. 3 , the recorded hologram is placed back at the plane where the recording was performed. The hologram is then illuminated from the right and the real conoscopic reconstruction forms at the place where the original object was placed. We can understand how this reconstruction takes place by considering how a single point $\mathrm{P}$ on the original object is first mapped to a FZL and then is refocused to a point in the system of fig. 3 . The light that upon reconstruction originates from each point on the hologram FZL, forms its own FZL at the reconstruction or object region. These FZL's add (incoherently) constructively only at $\mathrm{P}$. At points other than $\mathrm{P}$ in the reconstruction region, the FZL's add randomly and do not focus. The conoscopic hologram of a sin- 


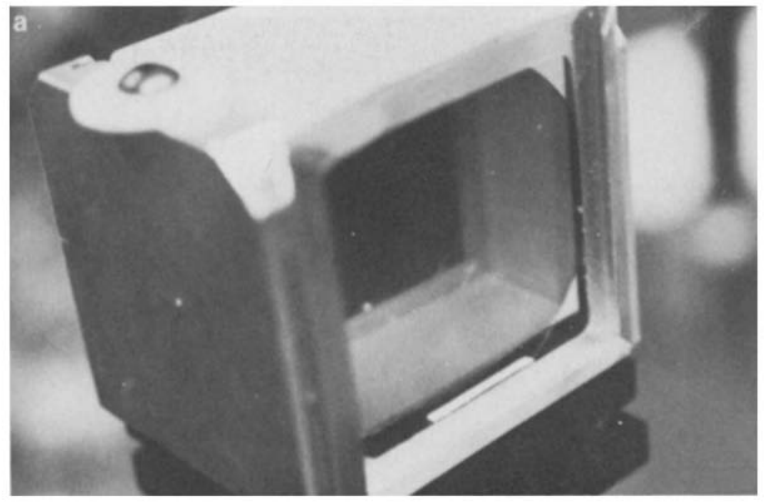

b

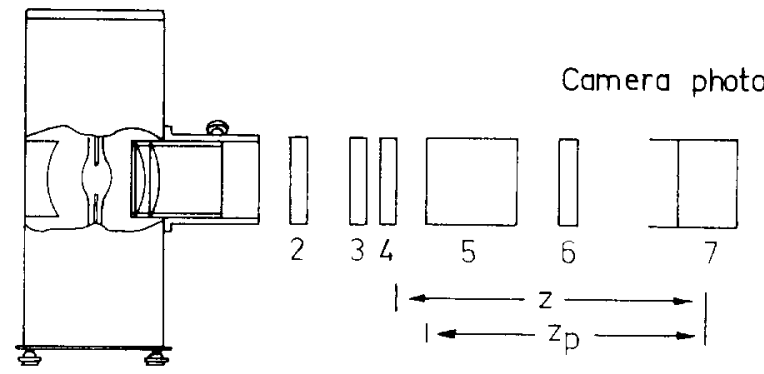

Fig. 5. Experimental system; a photography (a) and a schematic diagram (b).

gle point located at transverse coordinates $x, y$ can be written as

$$
\begin{aligned}
& H\left(x^{\prime}, y^{\prime}\right) \\
& \quad=\frac{1}{2}+\frac{1}{2} \cos \left[\left(\pi / \alpha z^{2}\right)\left(\left(x-x^{\prime}\right)^{2}+\left(y-y^{\prime}\right)^{2}\right)\right],
\end{aligned}
$$

where

$\alpha=\lambda / 2 L\left(n_{\mathrm{o}}-n_{\mathrm{e}}\right)$.

$x^{\prime}$ and $y^{\prime}$ are the spatial coordinates at the hologram plane, $\lambda$ is the wavelength of light, $n_{\mathrm{o}}$ and $n_{\mathrm{e}}$ are the two refractive indices, $L$ is the thickness of the crystal, and $z$ is the distance from the object to the hologram plane corrected for the geometrical effects of propagating through the crystal. The intensity at the reconstruction region (coordinates $\hat{x}, \hat{y}, \hat{z}$ ) is as follows [1]:

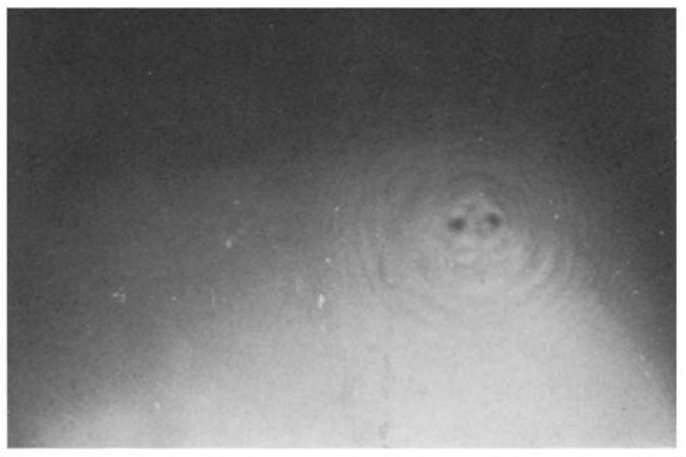

Fig. 6. Conoscopic reconstruction of the hologram of two points (fig. 4a).

$$
\begin{aligned}
& I(\hat{x}, \hat{y}, \hat{z}) \\
& \propto \int_{-\infty}^{+\infty} \int_{-\infty}^{+\infty} \cos \left[\left(\pi / \alpha z^{2}\right)\left(\left(x-x^{\prime}\right)^{2}+\left(y-y^{\prime}\right)^{2}\right)\right] \\
& \times \cos \left[\left(\pi / \alpha \hat{z}^{2}\right)\left(\left(\hat{x}-x^{\prime}\right)^{2}+\left(\hat{y}-y^{\prime}\right)^{2}\right)\right] \mathrm{d} x^{\prime} \mathrm{d} y^{\prime} \\
& + \text { bias. }
\end{aligned}
$$

Two functions $\cos \left[b\left(x-x_{1}\right)^{2}\right]$ and $\cos \left[b\left(x-x_{2}\right)^{2}\right]$ are orthogonal to each other if $\bar{b} \neq b$ and $x_{1} \neq x_{2}$. As a result the two dimensional integrand in eq. (2) collapses to a delta function at coordinates $x, y, z$.

Presented in fig. 6 are the results of a simple conoscopic reconstruction utilizing spatially incoherent light from a sodium lamp. In this case the object is comprised of two points and its conoscopic hologram consists of two superimposed FZL's. This hologram is shown in fig. $4 \mathrm{a}$. The two reconstructed points are very distinct in fig. 6 with good resolution and contrast. The resolution of the reconstruction is equal to the smallest fringe spacing (i.e. the spacing of the outermost ring in fig. 1) in the recorded hologram. The minimum resolution achievable is in practice limited by the resolution of the recording medium since the birefrigence and the thickness of available crystals can provide FZL's with very fine fringe spacing. The contrast of the conoscopic reconstruction is limited by the bias term that are unavoidably present in all incoherent reconstruction schemes. In the following section we consider reconstruction based on diffraction using coherent light instead of propagation in anisotropic crystals. This will allow the implementation of off-axis conoscopic holography leading to bias-free reconstruction. 


\section{Reconstruction of conoscopic holograms by diffraction}

A conoscopic hologram can also be reconstructed by illuminating it with a coherent beam and allowing the coherent field to propagate for a suitable distance. This will result in the reconstruction of the object because the transformation involved in the conoscopic hologram of a single point (eq. (1)) has strong similarities to the Fresnel diffraction pattern of a coherent point source. The conoscopic mapping is real having a cosinusoidal kernel instead of the complex exponential that is encountered in a Fresnel integral. This distinction is a consequence of the fact that in the incoherent conoscopic system we do not retain phase information while in a coherent system we do. A second difference between the conoscopic and the diffraction transformations is a different dependence with the longitudinal variable $z$. In a Fresnel integral the dependence is $1 / z$ in the exponent of the scattered spherical wave [2], whereas in the conoscopic transformation the dependence is $1 / z^{2}$ (see eq. (1)). When we use diffraction to reconstruct the conoscopic hologram, the second discrepancy will result in a nonlinear scaling of the $z$ axis. This will normally be a distortion in the reconstructed image and should be minimized by proper choice of the reconstructing parameters [1].

Fig. 7 shows the reconstruction that forms when the hologram of fig. $4 \mathrm{~b}$ is illuminated by a coherent plane wave. This reconstruction was obtained on axis. Therefore the undiffracted light (or DC component) and the conjugate image are superimposed on the reconstruction similarly to the conoscopic reconstruc-

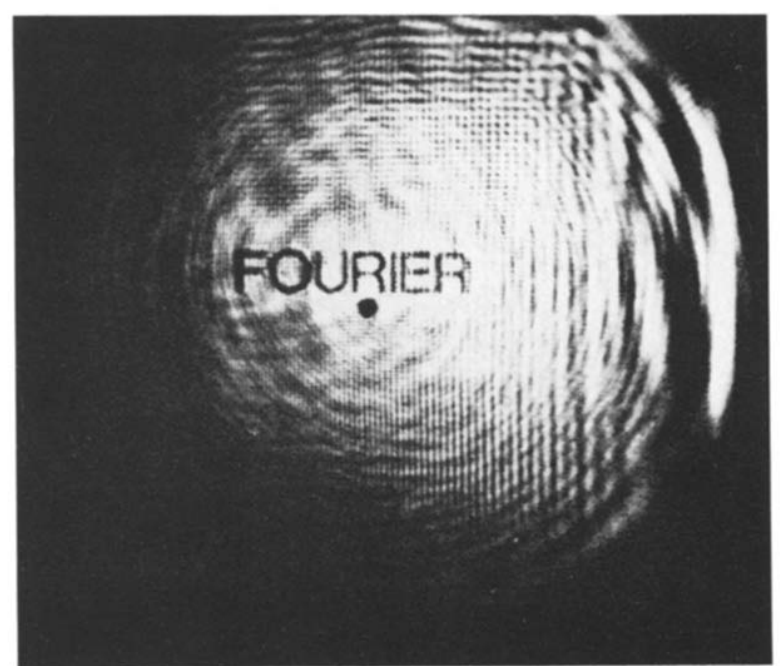

Fig. 7. Coherent reconstruction of the hologram of the word "FOURIER" ( fig. 4b).

tion. Comparing figs. 6 and 7 we notice that the effect of the bias is more pronounced in fig. 7 because of coherent effects. However the advantage of using coherent light is that it is possible to use spatial filtering to separate the bias from the reconstruction. To accomplish this we need to record an off-axis conoscopic hologram which is done simply by tilting the optical axis of the crystal relative to the axis perpendicular to the recording plane. The off-axis recording arrangement is shown in fig. 8. Let us consider what is the effect of tilting the crystal when the hologram of a single point target is formed. This is shown in fig. 9a. It is evident that the conoscopic figure has been translated and the center of the FZL

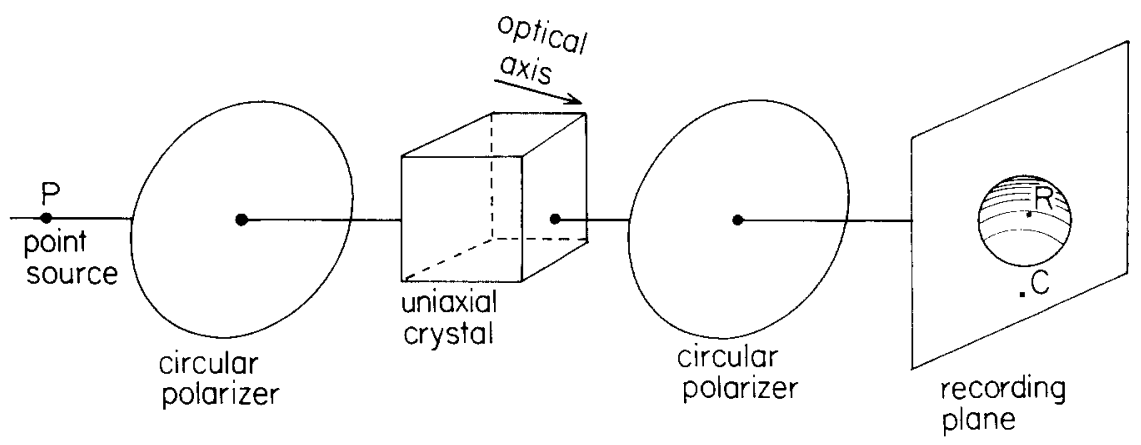

Fig. 8. Principle of off-axis conoscopic holography. 

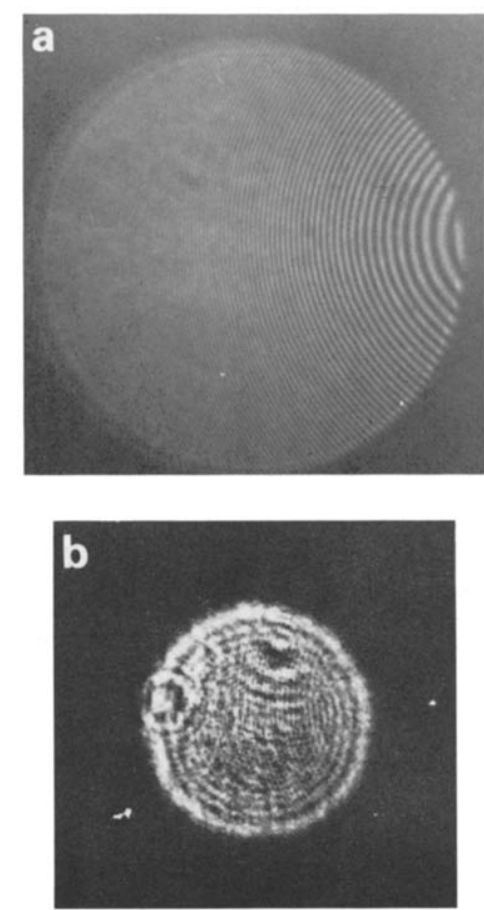

Fig. 9. Off-axis hologram (a), and coherent reconstruction (b) of a one point target.
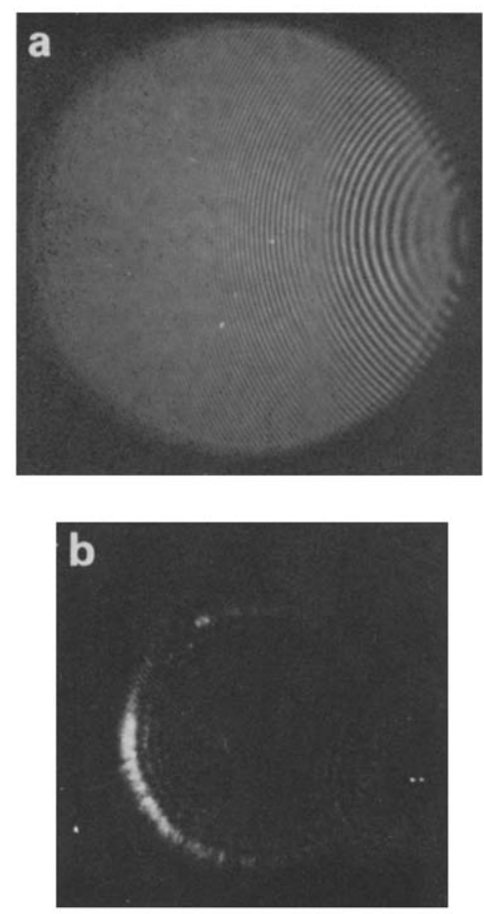

Fig. 10. Off-axis hologram (a) and coherent reconstruction (b) of a two-points target.
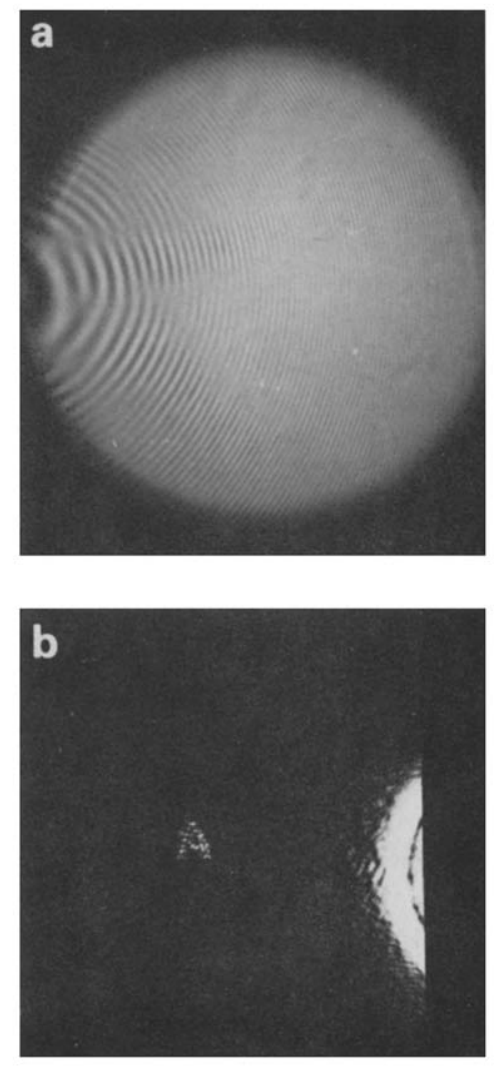

Fig. 11. Off-axis hologram (a) and coherent reconstruction (b) of letter "A".

has moved outside of the recording area. This is precisely analogous to the formation of a coherent hologram with an off-axis reference beam resulting in the formation of the hologram on a spatial carrier. This in turn allows us to separate the reconstructed image from the bias by spatial filtering, however the results image is high pass filtered. This is shown in fig. $9 \mathrm{~b}$ which is the coherent reconstruction of the hologram in fig. 9a. The large circular beam is the bias and the small, focused, spot to the right is the reconstruction. Figs. $10 \mathrm{a}$ and $10 \mathrm{~b}$ are the conoscopic hologram and reconstruction of two points and similarly fig. 11 shows the results when the letter " $\mathrm{A}$ " was used as the object.

A simple experiment was carried out in order to demonstrate the three dimensional imaging capability of this technique. Two planar transparencies of the words "FRESNEL" and "FOURIER" were prepared and placed at two different planes. An on-axis 

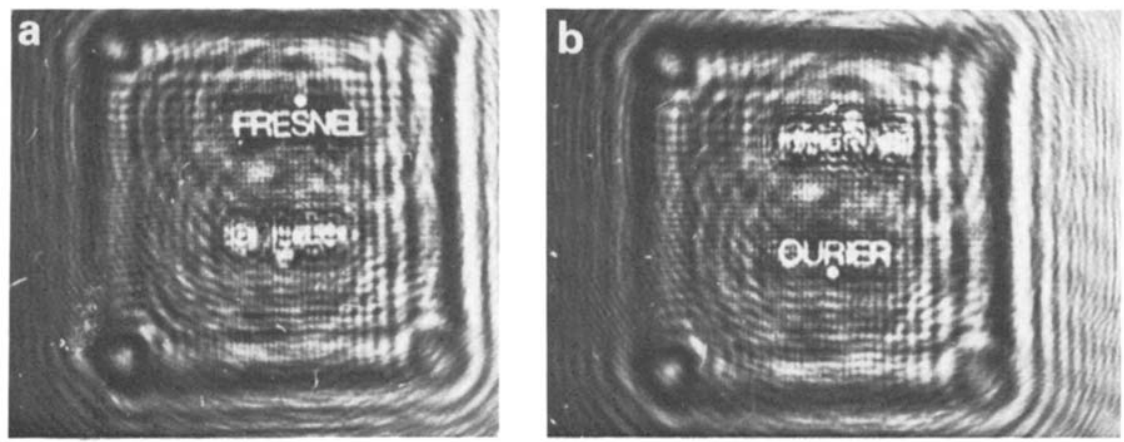

Fig. 12. Coherent reconstruction of two objects placed at two different planes.

conoscopic hologram of this object was recorded and reconstructed by diffraction. Figs. 12a and $12 \mathrm{~b}$ show the reconstruction at two different planes showing that the two images come to focus at two different regions in the three dimensional space.

\section{Computer reconstructions}

The conoscopic hologram can be formed on the photosensitive face of a television camera, digitized, and then operated upon with a digital computer which carries out the required transformation and forms the reconstruction. This technique has the appealing feature that it combines the simplicity of conoscopic optical recording with the flexibility of digital reconstruction. An experimental demonstration of this technique was carried out by first recording optically a conoscopic hologram of the word "FOURIER" on photographic film (the hologram of fig. 4b). The hologram was then digitized using a microdensitometer that was interfaced to a digital computer. The results of the computer reconstruction are shown in fig. 13. The reconstruction of fig. 13a was obtained by implementing a Fresnel integral, in other words the digital computer was used to simulate the optical reconstruction. Comparing figs. 13 a and 7 we see that they are virtually identical. Fig. $13 \mathrm{~b}$ was obtained by Wiener filtering [3] the reconstruction which smooths the coherent effects that are evident in the background bias in figs. 13a and 7. This example demonstrates the added flexibility that exists when the reconstruction is done digitally. We will address the algorithmic possibilities that exist in this framework in a forthcoming publication.

\section{Conclusion}

Three separate techniques were presented and experimentally demonstrated for the reconstruction of conoscopic holograms. The conoscopic reconstruction is very interesting, however, it may be limited
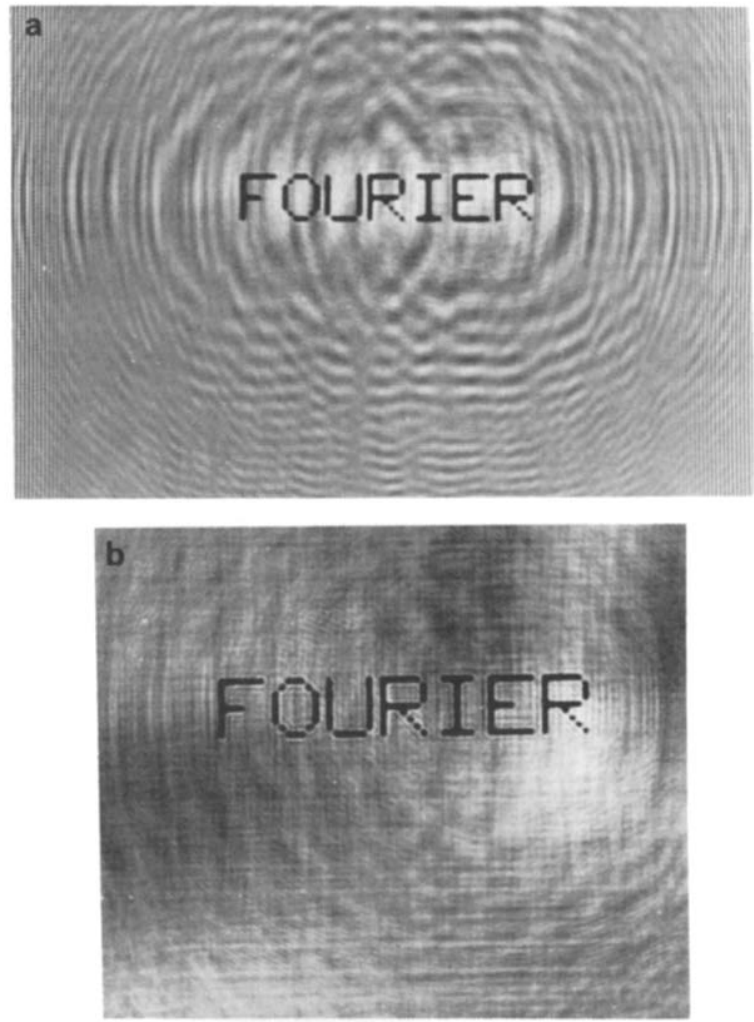

Fig. 13. Computer reconstruction of the hologram of the word "FOURIER" ( fig. 4b). 
as far as practical utility is concerned due to the bias problem. The diffraction based reconstruction permits the separation of the bias from the signal in an off-axis conoscopic hologram. The computer recontruction leads to maximum flexibility combined with the simplicity of conoscopic recording and appears to be well suited for applications such as 3-D machine vision.

\section{References}

[1] G. Sirat and D. Psaltis, Optics Lett. 10 (1985) 10.

[2] J.W. Goodman, Introduction to Fourier optics (McGrawHill, New York, 1968).

[3] W.K. Pratt, Digital image processing (Wiley Interscience, New York, 1978;. 